Supporting Information

\title{
Spinodally Decomposed Pbse-Pbte Nanoparticles for High- Performance Thermoelectrics: Enhanced Phonon Scattering and Unusual Transport Behavior
}

\author{
Min-Seok Kim, Woo-Jin Lee, Ki-Hyun Cho, Jae-Pyoung Ahn, and Yun-Mo Sung
}

\section{Calculating the electronic thermal conductivity $\left(\kappa_{E}\right)$}

The temperature-dependent $\kappa_{\mathrm{E}}$ values were estimated with the following equation. ${ }^{1,2}$

$$
\kappa_{E}=L \sigma T
$$

where $\mathrm{L}, \sigma$, and $\mathrm{T}$ are the Lorenz number, electrical conductivity, and absolute temperature, respectively. The $\sigma$ values were obtained from the experiments and the $\mathrm{L}$ values were calculated with the following equation: ${ }^{1,2}$

$$
\mathrm{L}=\left(\frac{k_{B}}{q}\right)^{2}\left\{\frac{\left(r+\frac{7}{2}\right) F_{r+\frac{5}{2}}(\eta)}{\left(r+\frac{3}{2}\right) F_{r+\frac{1}{2}}(\eta)}-\left[\frac{\left(r+\frac{5}{2}\right) F_{r+\frac{3}{2}}(\eta)}{\left(r+\frac{3}{2}\right) F_{r+\frac{1}{2}}(\eta)}\right]^{2}\right\}
$$

where $\mathrm{k}_{\mathrm{B}}, \mathrm{q}, \mathrm{r}$, and $\eta$ are the Boltzmann constant, charge, scattering exponent, and reduced chemical potential, respectively. The value of $r$ is assumed to be $-1 / 2$ (acoustic phonon scattering), although this value would deviate slightly at a high temperature above $523 \mathrm{~K}$. The $\eta$ values were estimated from the experimental data for the Seebeck (S) coefficient by using the following equation under the assumption of a single parabolic-band model with acoustic phonon scattering $(\mathrm{r}=-1 / 2):^{1,2}$

$$
\mathrm{S}=\frac{k_{B}}{q}\left[\eta-\frac{\left(r+\frac{5}{2}\right) F_{r+\frac{3}{2}}(\eta)}{\left(r+\frac{3}{2}\right) F_{r+\frac{1}{2}}(\eta)}\right]
$$

In the above equations, $F_{k}(\eta)$ represents the Fermi-Dirac function: 


$$
F_{k}(\eta)=\int_{0}^{\infty} \frac{x^{k}}{\exp (x-\eta)+1} d x
$$

The carrier concentration (n) of the bulk nanostructured $\mathrm{PbSe} / \mathrm{PbTe}$ was determined to be approximately $1.5 \times 10^{19} \mathrm{~cm}^{-3}$ (n-type) from the Hall measurements at room temperature, and this belongs to a degenerate regime. When we consider the different scattering modes, the uncertainty is $10-25 \%$.

\section{N-type thermoelectric (TE) characteristics according to the deficiency of Se and Te atoms}

The n-type conduction in the $\mathrm{PbSe} / \mathrm{PbTe} \mathrm{TE}$ samples without doping is due to the deficiency of Se and Te atoms. An excess of a chalcogen produces vacancies at the metal sites and leads to the formation of p-type TE materials, while an excess of $\mathrm{Pb}$ atoms generates an n-type TE material because of the lack of chalcogen atoms. ${ }^{3}$ This is described below in Kröger-Vink notation. ${ }^{4}$

$$
\begin{gathered}
\mathrm{Se}(\mathrm{Te})_{S e(T e)} \rightleftharpoons V_{S e(T e)}^{\times}+S e(T e) \\
V_{S e(T e)}^{\times} \rightleftharpoons V_{S e(T e)}^{*}+2 \mathrm{e}^{\prime}
\end{gathered}
$$

\section{Estimating the Seebeck coefficient (S) with a single Kane-band model}

The transport properties of a lead chalcogenide can be well described with a single Kaneband model. An in-depth explanation of this model is given in references. ${ }^{6,7}$ We estimated the temperature-dependent carrier density (n) with Hall measurements. The carrier densities of the $\mathrm{PbSe}$ and $\mathrm{PbSe} / \mathrm{PbTe}$ samples were determined to be $1.9 \times 10^{19}$ and $1.5 \times 10^{19} \mathrm{~cm}^{-3}$, respectively, and were relatively independent of temperature. Using the Kane band model, the transport parameters are expressed as follows. 
The carrier density (n):

$$
\mathrm{n}=\frac{\left(2 m^{*} k_{B} T\right)^{3 / 2}}{3 \pi^{2} \hbar^{3}} F_{0}^{3 / 2}
$$

and the generalized Fermi-Dirac integral $\left({ }^{n} F_{l}^{m}\right)$ :

$$
\begin{gathered}
{ }^{n} F_{l}^{m}(\eta, \varepsilon)=\int_{0}^{\infty}\left(-\frac{\partial f}{\partial \varepsilon}\right) \varepsilon^{n}\left(\varepsilon+\alpha \varepsilon^{2}\right)^{m}(1+2 \alpha \varepsilon)^{l} d \varepsilon \\
f=\frac{1}{e^{(\varepsilon-\eta)}+1} \text { and } \eta=\frac{\xi}{k_{B} T}
\end{gathered}
$$

where $\mathrm{k}_{\mathrm{B}}$ is the Boltzmann constant, $\hbar$ is the reduced Plank constant, $\mathrm{m}^{*}$ is the effective mass of the density of states when taking into account band degeneracy, $\varepsilon$ is the reduced energy, $\alpha$ is defined as $\alpha=\mathrm{k}_{\mathrm{B}} \mathrm{T} / \varepsilon_{\mathrm{g}}$, and $\varepsilon_{\mathrm{g}}$ is the band gap, which exhibits temperature dependence via $\mathrm{d} \varepsilon_{\mathrm{g}} / \mathrm{dT}=4 \times 10^{-4}$ in PbSe and PbTe. The effective mass also exhibits temperature dependence via $\mathrm{d} \ln \left(\mathrm{m}^{*}\right) / \mathrm{d} \ln (\mathrm{T})=0.5$ in both materials, with an in-depth list of physical properties presented in reference [6].

From the above calculations, the values for the reduced chemical potential $(\eta)$ were obtained for various temperatures. Using these values, the expected $S$ values were calculated using the following equation:

$$
\mathrm{S}=\frac{k_{B}}{e}\left[\frac{{ }^{1} F_{-2}^{1}}{{ }^{0} F_{-2}^{1}}-\eta\right]
$$

We also calculated the Lorenz number (L) using this model and concluded that the errors in this method are less than 5\%. We compared the calculated values to the experimental values, and the plot is depicted in Figure S5 of the Supporting Information.

\section{Possible effect of porosity}

The effect of porosity was considered using a Maxwell-Eucken expression. ${ }^{9}$

$$
X_{100}=X_{p} \frac{1+\beta P}{1-P}
$$


where $\mathrm{X}_{100}$ is the electrical or thermal conductivity when a sample has $100 \%$ density, $\mathrm{P}$ is the degree of porosity and $\beta$ is an empirical parameter related to the pore geometry. We assumed that the porosity exhibits spherical shape and is randomly distributed $(\beta=2)$. The densities of both $\mathrm{PbSe}$ and $\mathrm{PbSeTe}$ samples were determined to be approximately 96\%. The ZT values of both samples are not modified because the porosity would effect on both electrical and thermal conductivities. Porosity-correlated thermal conductivities are shown in Supporting Information Figure S9. 

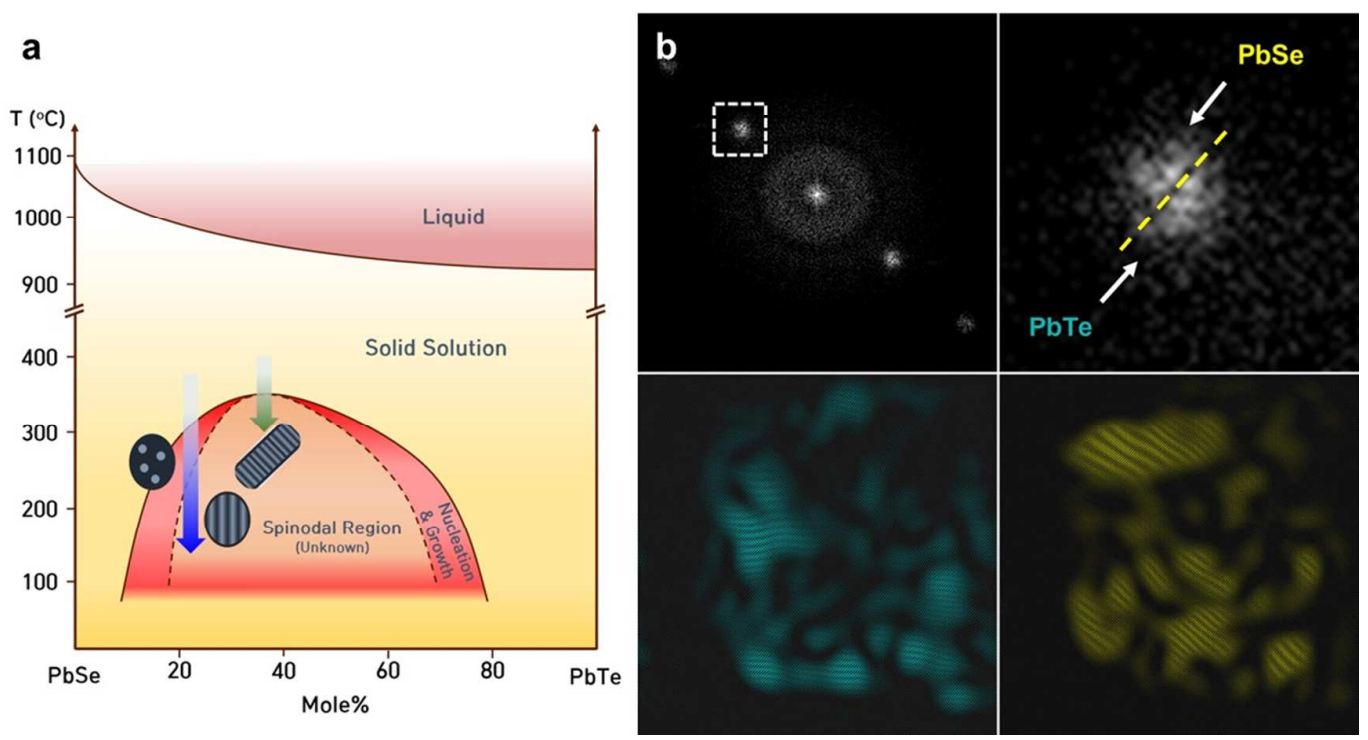

\section{Supplementary Figure 1. Spinodally decomposed PbSe/PbTe NPs.}

(a) Phase diagram for the PbSe and PbTe phases as functions of the temperature and molar ratio (Mole\%). This diagram was redrawn with the aid of reference [5]. PbSe and $\mathrm{PbTe}$ can initiate their respective nucleation and growth, spinodal decomposition, or solid-solution formation depending on the temperature and composition. (b) FFT patterns and their inverse images obtained from Figure 1c of the main text. The separated regions of $\mathrm{PbSe}$ and $\mathrm{PbTe}$ (top-right panel) from one spot (dashed rectangle in top-left panel) in the FFT patterns were identified. The inverse FFT (IFFT) images were extracted from this spot and are depicted in the bottom panels. The IFFT images clearly illustrate the local compositional fluctuations inside a single $\mathrm{PbSe} / \mathrm{PbTe} \mathrm{NP}$. 


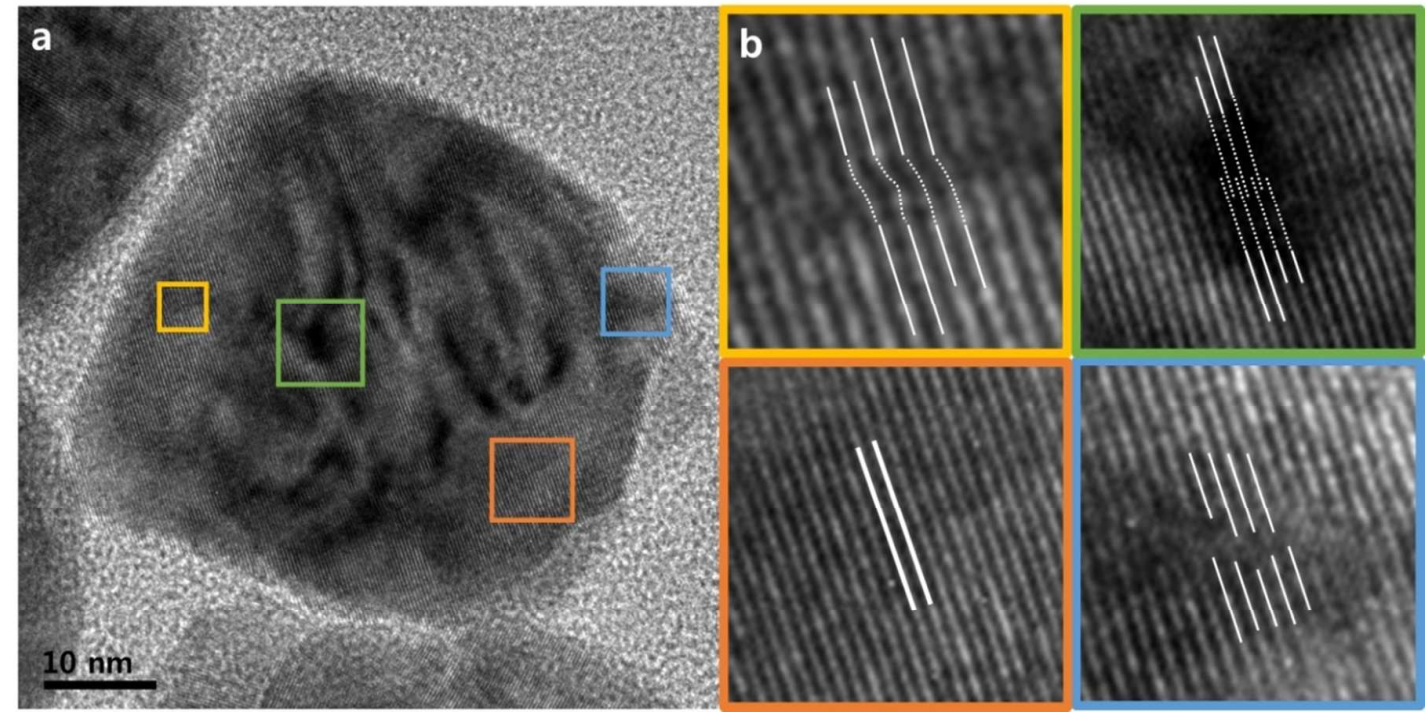

Supplementary Figure 2. High-magnification TEM images of a single PbSe/PbTe NP.

(a) A single NP manifesting continuous bands with contrast. (b) Line defects in the single NP shown in (a). The respective areas (marked with colored squares) of the single NP in (a) were magnified and the various line defects were identified (yellow $=$ curved, green $=$ staggered, orange $=$ no defects, blue $=$ disconnected). These defects are due to the phase fluctuations inside the single NP.

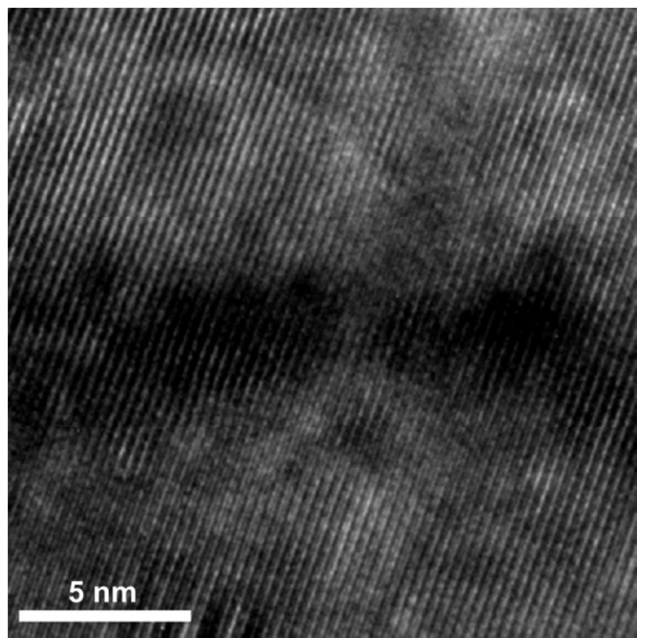

Supplementary Figure 3. Defects caused by excess Pb atoms.

A highly magnified image of Figure $2 \mathrm{c}$ of the main text. This region contains a few more $\mathrm{Pb}$ atoms and exhibits imperfections in the crystallinity or line defects. 


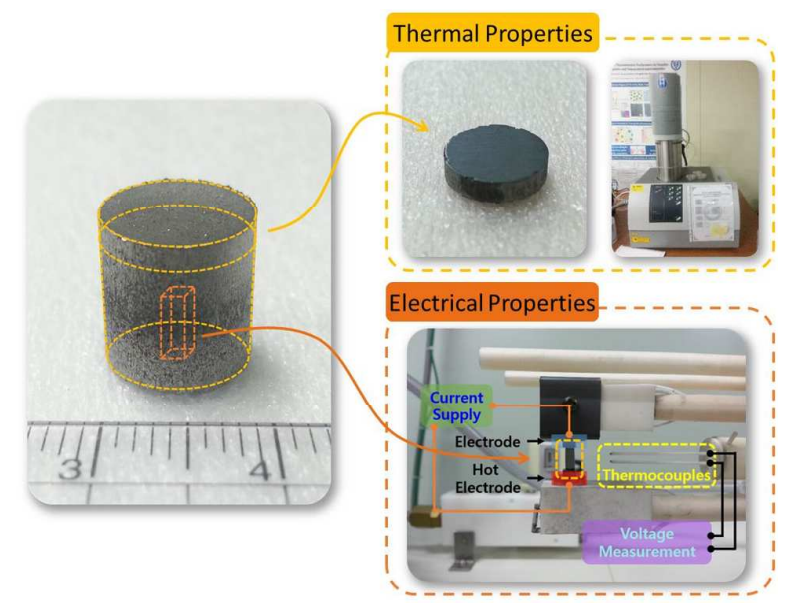

Supplementary Figure 4. Experimental setups used for the thermoelectric measurements.

The TE data for each cycle was acquired via a round robin test to prevent any possible inaccuracies that may arise from the characteristics of our measuring devices. To verify the isotropic nature of the properties, the same crystallographic orientations were chosen for each cycle.

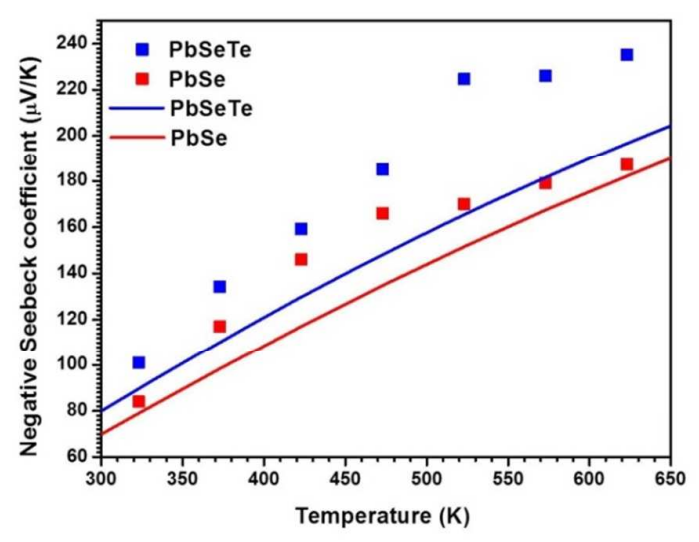

Supplementary Figure 5. Estimation of the Seebeck coefficient using a single Kane-band model.

The solid red and blue lines represent the estimated values for the $\mathrm{PbSe}$ and $\mathrm{PbSe} / \mathrm{PbTe}$ samples, respectively. We applied the Pisarenko relation to describe S vs. T. 

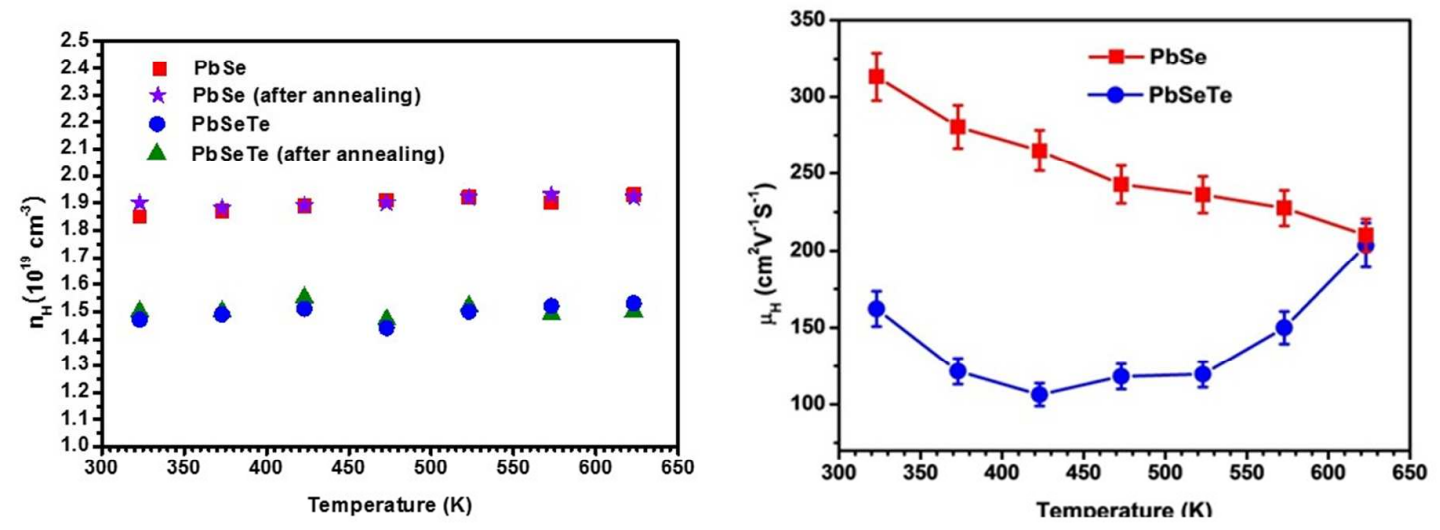

Supplementary Figure 6. Hall carrier densities and mobility of bulk PbSe and PbSeTe as a function of temperature.

The carrier densities of the $\mathrm{PbSe}$ and $\mathrm{PbSe} / \mathrm{PbTe}$ samples were found to be $1.9 \times 10^{19}$ and $1.5 \times 10^{19} \mathrm{~cm}^{-3}$, respectively, and these values are relatively independent of temperature. The mobility values also indicated and they exhibit the similar trend with the electrical conductivity as shown in Figure 4a of the main text.

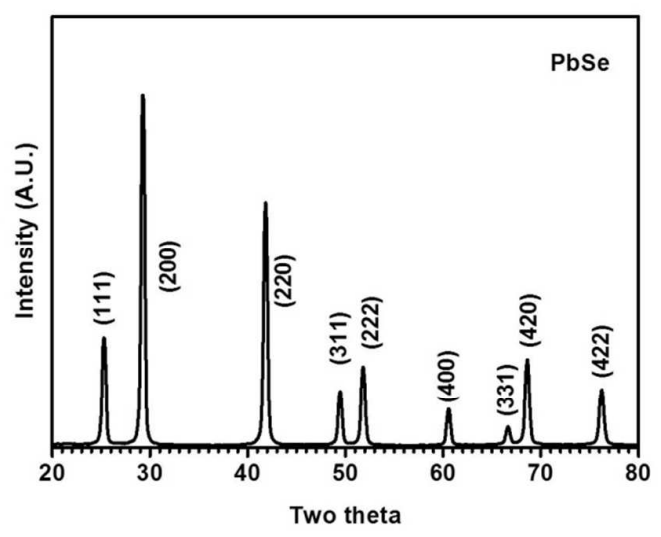

\section{Supplementary Figure 7. XRD pattern of the SPS-treated bulk PbSe sample.}

There is no $\mathrm{Pb}$ phase in the $\mathrm{XRD}$ pattern. In the $\mathrm{PbSeTe}$, the extra $\mathrm{Pb}$ phase was identified as shown in Figure 1a of the main text. 


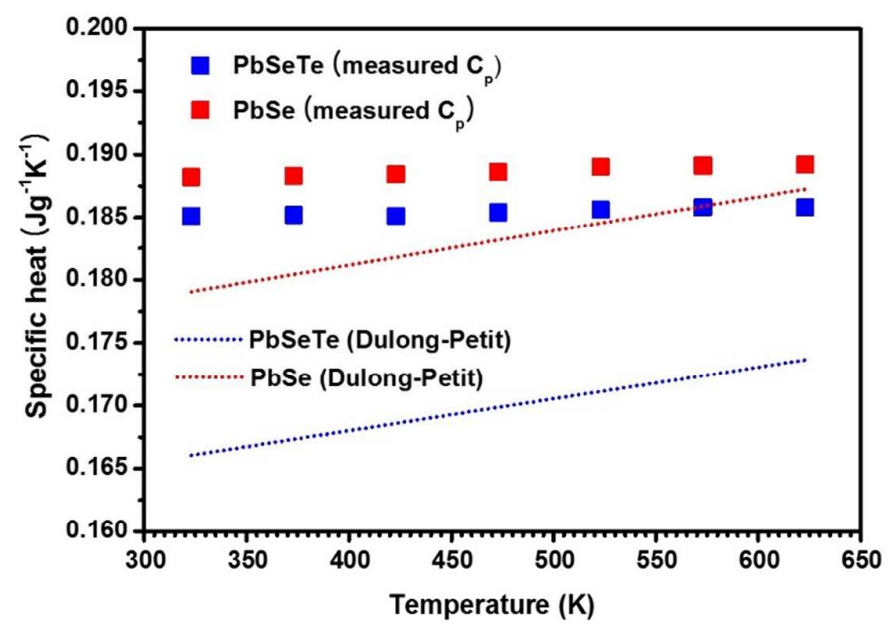

Supplementary Figure 8. Measured heat capacities of bulk PbSe and PbSeTe as a function of temperature

The heat capacities of bulk PbSe and PbSeTe were measured. In the case of PbSe, the heat capacity value exhibits the similar value with estimated one (dashed red line). However, $\mathrm{PbSeTe}$ exhibit higher heat capacity value than estimated one. Heat capacity values in materials having nanograins frequently exceeded the Dulong-Petit values because of large surface contribution. The estimation is obtained by using literature values in conjunction with the law of mixtures according to reference [8]. 


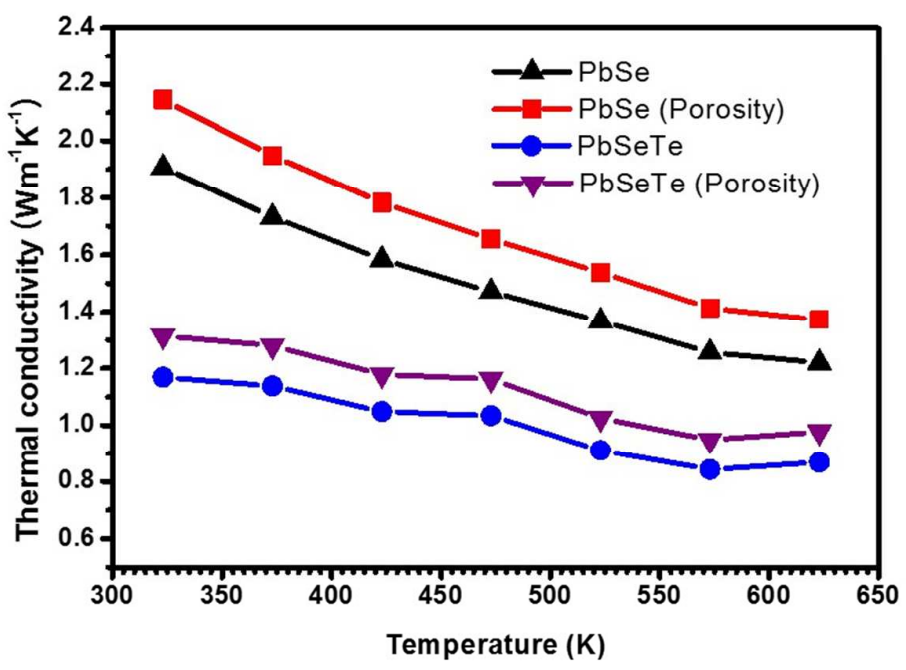

Supplementary Figure 9. Porosity-correlated thermal conductivity.

The ZT values of both samples are not modified because the porosity would effect on both electrical and thermal conductivities (main text). If the porosity affects the thermal conductivity only, the ZT values of $\mathrm{PbSe}$ and $\mathrm{PbSeTe}$ become 1.65 and 1.07, respectively.

\section{References}

1. Nolas G. S.; Sharp, J.; Goldsmid, H. J. Thermoelectrics: Basic Principles and New Materials Developments; Springer: Berlin, 2001; chap 2-3.

2. Biswas, K.; He, J.; Blum, I. D.; Wu, C.-I.; Hogan, T. P.; Seidman, D. N.; Dravid, V. P.; Kanatzidis, M. G. High-Performance Bulk Thermoelectrics with All-Scale Hierarchical Architectures. Nature 2012, 489, 414-418. 
3. Heremans, J. P.; Wiendlocha, B.; Chamoire, A. M. Resonant Levels in Bulk Thermoelectric Semiconductors. Energy Environ. Sci. 2012, 5, 5510-5530.

4. Walsh, A. Defect Processes in a PbS Metal Organic Framework: A Quantum-Confined Hybrid Semiconductor. J. Phys. Chem. Lett. 2010, 1, 1284-1287.

5. Liu, H.; Chang, L. L. Y. Phase Relations in the System PbS-PbSe-PbTe. Mineral. Mag. 1994, $58,567-578$.

6. Wang, H.; Schechtel, E.; Pei, Y.; Snyder, G. J. High Thermoelectric Efficiency of n-type PbS. Adv. Energy Mater. 2013, 3, 488-495.

7. Wang, H.; Pei, Y.; LaLonde, A. D.; Snyder G. J. Weak Electron-Phonon Coupling Contributing to High Thermoelectric Performance in n-type PbSe, Proc. Natl. Acad. Sci. U. S. A. 2012, 109, 9705-9709.

8. Blachnik, R.; Igel, R. Thermodynamic Properties of IV-VI Compounds Lead Chalcogenides. Naturforsch. B 1974, 29, 625-629.

9. Ibáñez, M.; Zamani, R.; Gorsse, S.; Fan, J.; Ortega, S.; Cadavid, D.; Morante, J. R.; Arbiol, J.; Cabot, A. Core-Shell Nanoparticles as Building Blocks for the Bottom-Up Production of Functional Nanocomposites: PbTe-PbS Thermoelectric Properties. ACS Nano, 2013, 7, 2573-2586. 